\title{
$\operatorname{CONF}-96 / 141--13$
}

\section{Local Field and Quantum Effects for Current Perpendicular to}

\section{Planes in Multilayers}

\author{
X.-G. Zhang \\ Computational Physics and Engineering Division,
}

Oak Ridge National Laboratory, P.O. Box 2008, Oak Ridge, Tennessee 37831-6114

W. H. Butler

Metals and Ceramics Division,

Oak Ridge National Laboratory, P.O. Box 2008, Oak Ridge, Tennessee 37831-6114

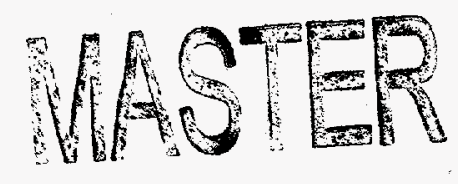

\section{Abstract}

The calculation of giant-magnetoresistance and in general, of electron transport for multilayers in the case of current perpendicular to the planes (CPP) requires both the two-point conductivity and the solution to the local field problem. In this paper we present a solution to the local field problem at an interface using two approaches. In the first approach we find the semiclassical solution for the local field when there is a band mismatch between two sides of an interface, and examine the deviation of the total resistance from the result of "self-averaging", in the lowest order of the value of the potential step. In the second approach, we solve for the quantum correction to the local field through a numerical iterative scheme. The oscillations due to the quantum correction are surprisingly large, but their correction to the total resistance is remarkably small. Our results imply that the "self-averaging" of the resistance, which is usually assumed in analysis of CPP, is only approximate. 


\section{DISCLAIMER}

Portions of this document may be illegible in electronic image products. Images are produced from the best available original document. 


\section{DISCLAIMER}

This report was prepared as an account of work sponsored by an agency of the United States Government. Neither the United States Government nor any agency thereof, nor any of their employees, make any warranty, express or implied, or assumes any legal liability or responsibility for the accuracy, completeness, or usefulness of any information, apparatus, product, or process disclosed, or represents that its use would not infringe privately owned rights. Reference herein to any specific commercial product, process, or service by trade name, trademark, manufacturer, or otherwise does not necessarily constitute or imply its endorsement, recommendation, or favoring by the United States Government or any agency thereof. The views and opinions of authors expressed herein do not necessarily state or reflect those of the United States Government or any agency thereof. 
The study of the giant magnetoresistance effect ${ }^{1}$ in magnetic multilayers has often relied on semiclassical models ${ }^{2,3}$. In the absence of band mismatches between different layers the semiclassical approach was shown ${ }^{4}$ to be quite accurate when the current direction is parallel to the layers (current in plane, or CIP). The semiclassical approximation is also believed to be accurate for the case of current perpendicular to the planes (CPP) when no band mismatches exist. It is the purpose of this paper to investigate whether any corrections are needed when there are band mismatches (or equivalently, potential steps) between layers for the CPP case.

Theoretical study of transport for CPP in a multilayer system requires the solution of the local field problem in addition to the calculation of the two-point conductivity. Unlike the case of current in the planes (CIP) where the electric field is uniform and only the conductivity is need to calculate the current, in the case of CPP the field is inhomogeneous due to the inhomogeneity of the system. The solution of the local field correction can be found from the current continuity condition,

$$
J=\int_{-\infty}^{\infty} d z^{\prime} \sigma\left(z, z^{\prime}\right) E\left(z^{\prime}\right),
$$

where $E$ is the local field, $\sigma$ is the two-point conductivity, and $J$ is the current density whose independence of $z$ puts a constraint on $E$.

In a semiclassical theory ${ }^{2,3}$, the local field is determined by the local scattering rate. When there is no band mismatch, and if the scattering rate is uniform within a layer, the local field can be shown to be a constant in this layer,

$$
E(z) \propto \frac{1}{\lambda(z)}
$$

where the local mean free path $\lambda(z)$ corresponds to the scattering rate at the layer $z$. This solution is also obtained quantum mechanically in the limit of thick layers, i.e. $d \gg \lambda$, where $d$ is the layer thickness. In the thin limit when $d \ll \lambda$, it can be shown ${ }^{4}$ from the quantum solution that the scattering rate is self-averaging. These results led to the general belief that the resistance is always self-averaging for current perpendicular to the layers. 
The first step in calculating the local field correction is to find the two-point conductivity. To this end we apply the the Kubo-Greenwood linear response theory ${ }^{5,6}$. We describe the potential step at the interface with the parameter $v$ as defined in $v^{2}=k_{1 F}^{2}-k_{2 F}^{2}$. The Green function for a single interface, where the self-energy changes abruptly, can be shown to be,

$$
\frac{\hbar^{2}}{2 m} G\left(z, z^{\prime}\right)= \begin{cases}\frac{1}{2 i k_{1}}\left[e^{i k_{1}\left|z-z^{\prime}\right|}+f e^{-i k_{1}\left(z+z^{\prime}\right)}\right], & z<0 \text { and } z^{\prime}<0 \\ \frac{1}{i\left(k_{1}+k_{2}\right)} e^{i\left(k_{2} z^{\prime}-k_{1} z\right)}, & z<0<z^{\prime},\end{cases}
$$

where $f=\left(k_{1}-k_{2}\right) /\left(k_{1}+k_{2}\right)$, and $k_{1}$ and $k_{2}$ are the $z$ components of the wavevectors on two sides of the interface. The full quantum solution for the conductivity is very complex. We will present a numerical solution in the second half of this paper. For now we apply the semiclassical approximation $\left(k_{i F} \lambda_{i} \gg 1\right.$ where $i$ labels layer) to the Kubo-Greenwood formula, and the semiclassical conductivity for CPP is given by

$$
\sigma_{\mathrm{sc}}\left(z, z^{\prime}\right)=\frac{e^{2}}{4 \pi^{2} \hbar}\left[k_{i F}^{2} E_{3}\left(\frac{\left|z-z^{\prime}\right|}{\lambda_{i}}\right)-\int_{0}^{\infty} k_{\|} d k_{\|}|r|^{2} e^{-2 k_{i I}\left|z+z^{\prime}\right|}\right],
$$

for $z$ and $z^{\prime}$ at the same side of the interface, where $i=1,2$ denotes the regions on both sides of the interface, and $r$ is the reflection coefficient,

$$
r=\frac{k_{1}-k_{2}}{k_{1}+k_{2}}
$$

For $z<0<z^{\prime}$, we get

$$
\sigma_{\mathrm{sc}}\left(z, z^{\prime}\right)=\frac{e^{2}}{4 \pi^{2} \hbar} \int_{0}^{\infty} k_{\|} d k_{\|}\left(1-|r|^{2}\right) e^{-2\left|k_{2} z^{\prime}-k_{11} z\right|} .
$$

Far away from the interface one gets,

$$
\sigma_{\mathrm{sc}}\left(z, z^{\prime}\right)=\frac{e^{2} k_{i F}^{2}}{4 \pi^{2} \hbar} E_{3}\left(\frac{\left|z-z^{\prime}\right|}{\lambda_{i}}\right) .
$$

In the above equations the terms containing $|r|^{2}$ can be identified as due to reflection on the interface. Although it is second order in $v$ it cannot be neglected because the contact resistance due to the interface is an even function of $v$ and thus is also second order.

The algebra for the local field correction, even in the semiclassical limit, is rather involved. We present here the result for the correction to the resistance when the potential step is 
small. If we drive a constant current $J$ through the interface, then the change in the voltage drop due to the interface contact resistance $\Delta V$ is given by,

$$
\frac{\Delta V}{J}=\frac{24 \pi^{2} \hbar}{e^{2}} \frac{\bar{v}^{2}}{k_{1 F}^{2}+k_{2 F}^{2}}\left(A_{1}+A_{2}\right)
$$

where we find the approximations to $A_{1}$ and $A_{2}$ by fitting to numerical solutions of the integrals involved in $A_{1}$ and $A_{2}$,

$$
\frac{1}{k_{b}^{4}} \frac{2 t_{0}}{1+2 t_{0}^{2} / k_{b} \lambda_{a}+13.4 t_{0}^{3}|v|^{\frac{3}{2}} / k_{b}^{3}+2 t_{0}^{4}\left(6.4+13 \sqrt{|v|} t_{0} / k_{b}+7.5|v| t_{0}^{2} / k_{b}^{2}\right) / k_{b}^{2} \lambda_{a}^{2}},
$$

and

$$
A_{2}=\frac{d_{b}}{k_{b}^{4}}
$$

Here we used the following symbols, $\bar{v}^{2}=v^{2}+\left(k_{1 F} / \lambda_{1}-k_{2 F} / \lambda_{2}\right)^{2}, t_{0}=\sqrt{\frac{2 k_{1 F} k_{2 F}+k_{2 F}^{2}}{2|v|}+1}$, $k_{a}^{2}=\left(k_{1 F}+k_{2 F}\right)^{2}+2|v|, d_{a}=\frac{3 \pi}{16} \sqrt{2 k_{a} \lambda_{a}}-1, \lambda_{a}=\lambda_{1} \sqrt{k_{1 F} / k_{2 F}}, k_{b}^{2}=\left(k_{1 F}+k_{2 F}\right)^{2}-2|v|$, $d_{b}=\frac{3 \pi}{16} \sqrt{2 k_{b} \lambda_{b}}-1$, and $\lambda_{b}=\lambda_{2} \sqrt{k_{2 F} / k_{1 F}}$.

In the semiclassical approach of Camblong-Levy ${ }^{2,3}$, the small difference in the Fermi wavevectors on both sides of the interface due to the difference in the scattering rates is neglected. If we take a semiclassical limit that is consistent with this approach, we must take $k_{i F} \lambda_{i}$ to be greater than all other quantities (such as $k_{1 F} / \sqrt{|v|}$ ). In this limit one gets,

$$
\frac{\Delta V}{J}=\frac{9 \pi^{3} \hbar}{2 e^{2}} \frac{v^{2}}{k_{1 F}^{2}+k_{2 F}^{2}} \frac{\sqrt{2 k_{b} \lambda_{b}}}{k_{b}^{4}}
$$

In Table I we compare the results of Eq. (8), Eq. (11) and those from numerical calculations using the full quantum two-point conductivity. There is some uncertainty in the accuracy of the numerical results due to the difficulty of converging the calculation for small potential steps. However, the agreement between Eq. (8) and the numerical results is good. The "true" semiclassical results from Eq. (11) are significantly below the numerical solutions for large potential steps. In the last column we also list the results given by the formula in Ref. ${ }^{7}$, which in the limit of an interface between two semi-infinite systems is, 


$$
\frac{\Delta V}{J}=0.35 \frac{\pi \hbar}{e^{2}} \frac{\left(k_{1 F}-k_{2 F}\right)^{2}}{k_{1 F}^{2} k_{2 F}^{2}}
$$

It gives results that are at least two orders of magnitude too small.

Because of the complex form of the quantum conductivity, it is not possible to derive an analytic solution for the local field correction. We used an iterative numerical approach that successively refined the local field according to the error in the current calculated from Eq. (1). The results were surprising. We found very large amplitudes for the local field oscillations. The amplitude decays slowly away from the interface, with characteristic lengths much greater than the mean-free-path. We also found that the period of the oscillation is strongly correlated with the Fermi wavelength and the mean-free-path. Finally, the correction to the total voltage drop across the interface is very small. For the cases we solved this extra voltage drop corresponds to a resistance equivalent to an added layer with thicknesses ranging from less than $1 \AA$ up to about $5 \AA$. This correction is negligible in most applications but can be significant in very thin films. Fig. 1 shows a typical spatial distribution of the local field $E(z)$ calculated for an interface where there is no band mismatch and the local effective mean-free-paths are $\lambda=75.6 \AA$ for $z<0$ and $\lambda=94.5 \AA$ for $z>0$.

In Table II we list the wavevectors of the local field oscillation on both sides of the interface for various interface configurations. We find that the wavevector depends only on the local scattering rate and the Fermi wavevector, and does not depend on the parameters on the other side of the interface. When these data are plotted in terms of a dimensionless function $q / k_{F}$ where $q$ is the wavevector of the oscillation as a function of the dimensionless variable $k_{F} \lambda$, we find that all the points fall on a universal curve described by,

$$
\frac{q}{k_{F}}=0.141+\frac{1.64}{\sqrt{k_{F} \lambda+2.4}} .
$$

The fit is shown in Fig.2.

Our results show that the wavelength of the local field oscillation is usually longer than the Fermi wavelength. When the mean-free-path is long, the local field is approximately uniform over the distance of a Fermi wavelength. This provides the justification for the 
approximation made in recent first-principles calculations ${ }^{8}$ of GMR that the local field is a constant within an atomic cell.

This work was supported by the Department of Energy Defense Programs, Assistant Secretary, Technology Management Group, Technology Transfer Initiative under under contract DE-AC05-96OR22464 with Lockheed Martin Energy Research Corporation. 


\section{TABLES}

TABLE I. Comparison of the predictions of the resistance per unit area at the interface from Eq. (8), where terms proportional to $v$ (the step in the real part of the potential) and $\Delta_{i}$ (the scattering rates) are all kept, but the semiclassical two-point conductivity is used, and from Eq. (11), where $v \gg \Delta_{i}$ is assumed. Calculated resistance from full quantum two-point conductivity are also presented. The last column is the prediction by the formula given in Ref. ${ }^{7}$. In all cases $E_{F}-V_{2}=0.26$ Hartree.

\begin{tabular}{ccccccc}
\hline \hline$\lambda_{1}$ & $\lambda_{2}$ & $E_{F}-V_{1}$ & $\begin{array}{c}\text { Quantum } \\
\left(h / e^{2}\right)\end{array}$ & $\begin{array}{c}\text { Eq. (8) } \\
\left(h / e^{2}\right)\end{array}$ & $\begin{array}{c}\text { Eq. (11) } \\
\left(h / e^{2}\right)\end{array}$ & $\begin{array}{c}\text { Ref. }^{7} \\
\left(h / e^{2}\right)\end{array}$ \\
\hline 75.6 & 94.5 & 0.25 & $6 \times 10^{-5}$ & $5.5 \times 10^{-5}$ & $3.2 \times 10^{-5}$ & $1.8 \times 10^{-7}$ \\
75.6 & 94.5 & 0.16 & $8 \times 10^{-3}$ & $8.3 \times 10^{-3}$ & $8.4 \times 10^{-3}$ & $3.6 \times 10^{-5}$ \\
37.8 & 75.6 & 0.25 & $5 \times 10^{-5}$ & $5.1 \times 10^{-5}$ & $2.3 \times 10^{-5}$ & $1.8 \times 10^{-7}$ \\
37.8 & 75.6 & 0.16 & $7 \times 10^{-3}$ & $5.8 \times 10^{-3}$ & $5.9 \times 10^{-3}$ & $3.6 \times 10^{-5}$ \\
\hline \hline
\end{tabular}


TABLE II. The wavevectors of the quantum oscillation in the local field. $E_{F}-V$ are in Hartrees and all other numbers in atomic units. Each row corresponds to an interface system where the wavevectors for the two sides are under the columns labeled with the corresponding mean free paths.

\begin{tabular}{ccccc}
\hline \hline$E_{F}-V_{1} \mid E_{F}-V_{2}$ & $\lambda=10$ & $\lambda=20$ & $\lambda=40$ & $\lambda=50$ \\
\hline $0.26 \mid 0.26$ & 0.4816 & 0.3897 & \\
$0.26 \mid 0.26$ & 0.4816 & & 0.3136 & \\
$0.26 \mid 0.26$ & 0.4816 & & & \\
$0.26 \mid 0.26$ & & 0.3897 & 0.3135 & \\
$0.26 \mid 0.26$ & & & 0.3135 & 0.2920 \\
\hline $0.13 \mid 0.13$ & 0.3778 & 0.3066 & & \\
$0.13 \mid 0.13$ & 0.3779 & & 0.2474 & \\
$0.13 \mid 0.13$ & 0.3779 & & & 0.2306 \\
$0.13 \mid 0.13$ & & 0.3066 & 0.2474 & \\
$0.13 \mid 0.13$ & & & 0.2473 & 0.2306 \\
\hline $0.25 \mid 0.26$ & & 0.3842 & 0.3135 & 0.2920 \\
$0.16 \mid 0.26$ & & 0.3296 & 0.3095 & \\
$0.25 \mid 0.26$ & & & 0.2657 & \\
$0.16 \mid 0.26$ & & & & \\
\hline \hline
\end{tabular}




\section{FIGURES}

FIG. 1. A typical local field calculated numerically from the current continuity condition. The local effective mean-free-paths are $\lambda=75.6 \AA$ for $z<0$ and $\lambda=94.5 \AA$ for $z>0$. The semiclassical theory of Camblong and Levy predicts a constant local field of $E=0.02403$ for $z<0$ and a constant field of $E=0.01922$ for $z>0$.

FIG. 2. Calculated wavevectors of the local field oscillation from various interfaces. The solid curve is Eq.(13). 


\section{REFERENCES}

${ }^{1}$ M. N. Baibich, J. M. Broto, A. Fert, F. Nguyen Van Dau, F. Petroff, P. Eitenne, G. Creuzet, A. Friederich, and J. Chazelas, Phys. Rev. Lett. 61, 2472 (1988).

${ }^{2}$ H. E. Camblong and P. M. Levy, Phys. Rev. Lett. 69, 2835 (1992); H. E. Camblong and P. M. Levy, J. Appl. Phys. 73, 5533 (1993).

${ }^{3}$ H. E. Camblong, Phys. Rev. B 51, 1855 (1995).

${ }^{4}$ X.-G. Zhang and W. H. Butler, Phys. Rev. B 51, 10085 (1995).

${ }^{5}$ R. Kubo, J. Phys. Soc. Jpn. 12, 570 (1957).

${ }^{6}$ D. A. Greenwood, Proc. Phys. Soc. London 71, 585 (1958).

${ }^{7}$ A. Vedyaev, C. Cowache, N. Rizhanova, and B. Dieny, Phys. Lett. A198, 267 (1995).

${ }^{8}$ W. H. Butler, X.-G. Zhang, D. M. C. Nicholson, and J. M. MacLaren, Phys. Rev. B52, 13399 (1995). 


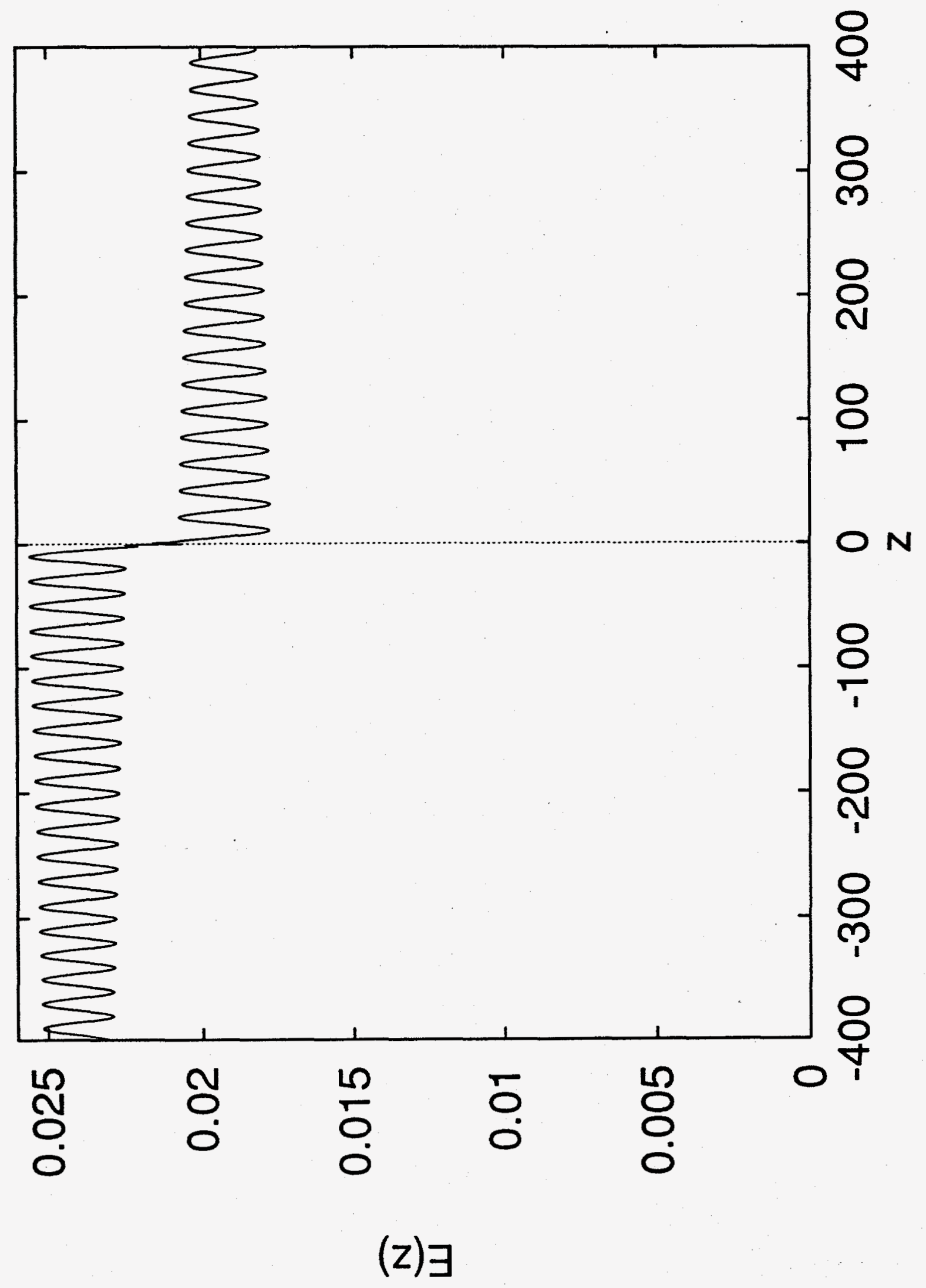




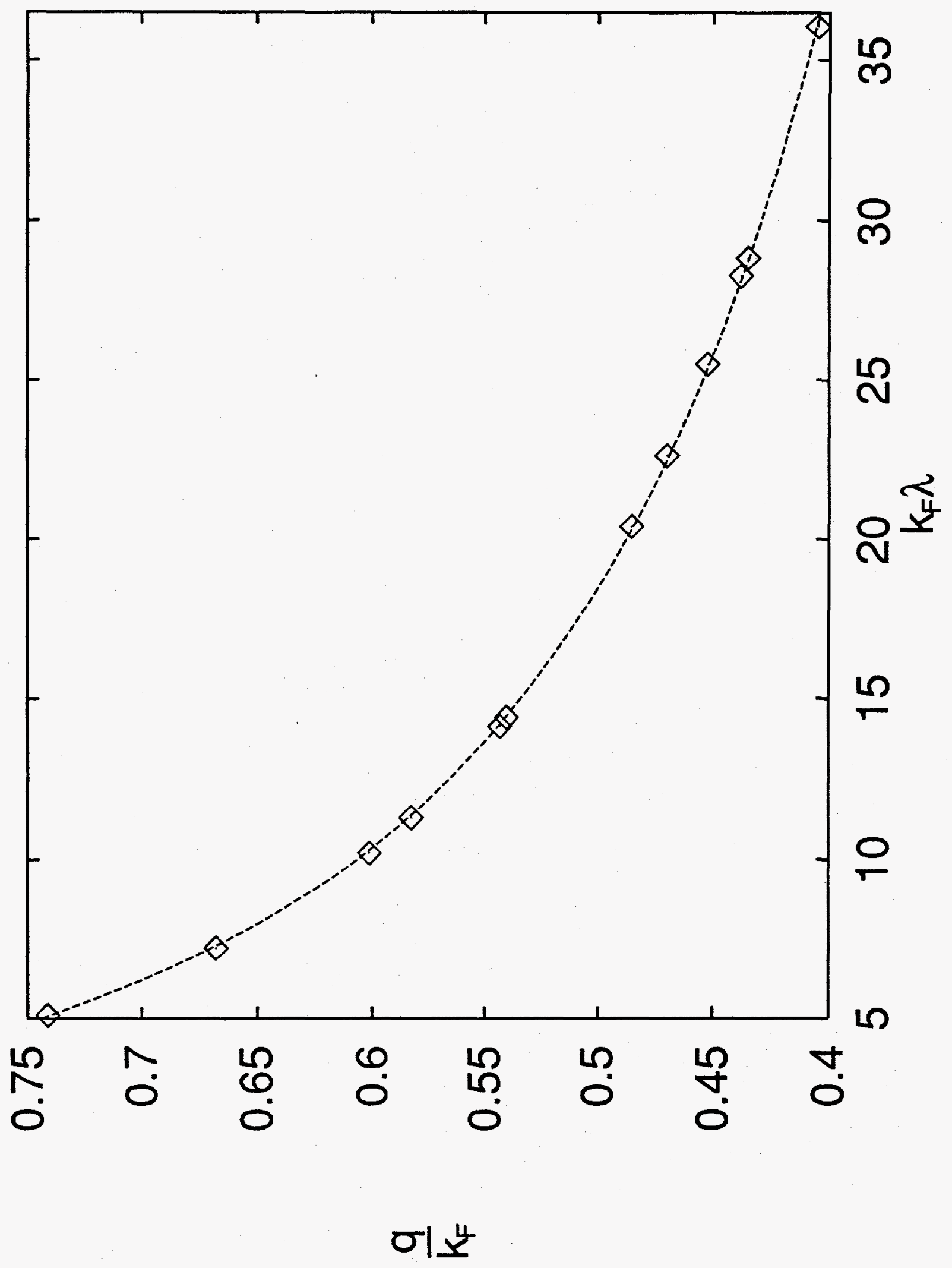

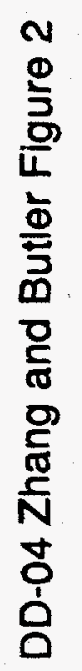

